\title{
Qualidade microbiológica de queijos tipo Minas comercializados em Belo Horizonte, MG, Brasil
}

\author{
Microbiological quality of Minas type cheeses marketed in Belo \\ Horizonte, $M G$, Brazil
}

Recebido em: 09/03/2020 Aceito em: $01 / 08 / 2020$
Vanessa Cristina LOPES $^{1}$; Elika Karina GUEDES ${ }^{1}$; Marcus Vinicius Cossenzo CANDIOTO ${ }^{1}$; Fernanda Meneghello DELVIVO $^{1}$; Adriene Ribeiro LIMA ${ }^{2}$ ${ }^{1}$ Centro Universitário UNA. Rua dos Guajajaras, 175, Centro, CEP 30180-100. Belo Horizonte, $M G$, Brasil. ${ }^{2}$ Faculdade de Farmácia, Universidade Federal Fluminense. Rua Dr. Mario Vianna, 523, Santa Rosa, CEP 24241-000. Niterói, RJ, Brasil. E-mail: adrienelima@id.uff.br

\section{ABSTRACT}

This study evaluated the microbiological quality of Minas cheeses commercialized in Belo Horizonte, MG, Brazil. We used eighteen cheese samples, nine of industrialized Minas frescal type marketed on supermarkets, and nine of the Minas artisanal type marketed in the County market. The temperatures of the cheese samples were measured during the exposure phase for sale. The hygienic-sanitary quality of cheeses was evaluated by counting coliforms at $45^{\circ} \mathrm{C}$, coagulase-positive Staphylococcus, and Salmonella sp. It was verified that eight (88.9\%) samples of industrialized Minas frescal cheese were at temperatures higher than allowed at the retail market, and $100 \%$ of the artisanal samples were exposed in inadequate temperatures. Two (22\%) samples of industrialized Minas cheese and four (44\%) samples of artisanal cheeses presented higher counts than those recommended by the legislation for coliforms at $45{ }^{\circ} \mathrm{C}$. Concerning the coagulasepositive Staphylococcus, $100 \%$ of the samples of both, industrialized and artisanal cheese, presented a higher amount than the legal standards. All analyzed samples showed contamination by Salmonella sp. It was concluded that the hygienic-sanitary quality of the 100\% Minas cheese evaluated was unsatisfactory and not safe for use.

Keywords: Staphylococcus aureus; Salmonella sp.; thermotolerant coliforms.

\section{RESUMO}

O objetivo deste estudo foi avaliar a qualidade microbiológica de queijos Minas industrializados e artesanais comercializados em Belo Horizonte, MG. Foram coletadas 18 amostras de queijos, sendo nove do tipo Minas frescal, industrializados comercializados em supermercados; e nove do tipo Minas artesanal, comercializados no Mercado Municipal. A temperatura dos queijos amostrados foi aferida durante a fase de exposição para venda. A qualidade higiênico-sanitária dos queijos foi avaliada por meio da contagem 
de coliformes a $45{ }^{\circ} \mathrm{C}$, Staphylococcus coagulase-positiva e pesquisa de Salmonella sp. Oito (88,9 \%) amostras de queijo Minas frescal industrializado se encontravam em temperaturas acima do permitido no mercado varejista, e $100 \%$ das amostras de queijo artesanal estavam expostas a temperaturas inadequadas nas lojas do Mercado Municipal. Duas (22\%) amostras de queijo Minas industrializado e quatro (44 \%) amostras de queijo Minas artesanal apresentaram contagens superiores ao preconizado pela legislação para coliformes a $45^{\circ} \mathrm{C}$. Em relação à contagem de Staphylococcus coagulase-positiva $100 \%$ das amostras, tanto de queijo industrializado quanto de queijo artesanal, apresentaram contagens superiores aos padrões legais. Nenhuma amostra apresentou contaminação pela bactéria Salmonella sp. Dessa forma, a qualidade higiênico-sanitária de $100 \%$ dos queijos Minas avaliados foi insatisfatória.

Palavras-chave: Staphylococcus aureus; Salmonella sp.; coliformes termotolerantes.

\section{INTRODUÇÃO}

O queijo é uma das formas mais antigas de alimento manufaturado que, junto de sua matéria prima, o leite, permitiram que a população sobrevivesse a fases de muitas necessidades. Por ser de fácil aceitação, o queijo é um alimento de grande comercialização, bem distribuído e apresenta alto rendimento na fabricação (1). É considerado um alimento de alto valor nutricional, fonte proteica de alta qualidade, alto teor de gorduras, cálcio, além de vitaminas e outros sais minerais (2).

O queijo Minas é um dos queijos brasileiros mais populares. Em Minas Gerais, seu Estado de origem, apresenta forte apelo histórico, cultural e turístico e, consequentemente, também econômico e social, sendo consumido por todas as camadas da população $(3,4)$. Sua fabricação é obtida por coagulação enzimática do leite pasteurizado ou cru a partir da adição do coalho, complementada ou não com ação de bactérias lácticas específicas $(3,5,6)$.

É um queijo com sabor suave ou levemente ácido, sem crosta e altamente perecível, pois além de apresentar alto teor de umidade, é rico em nutrientes que o torna bastante suscetível a contaminação microbiana. Destacam-se, entre os contaminantes de queijos, bactérias causadoras de doenças alimentares como Staphylococcus aureus e Salmonella sp., além de bactérias do grupo dos coliformes a $45{ }^{\circ} \mathrm{C}$ (coliformes termotolerantes), que são indicadores de contaminação e, se as contagens são altas, podem indicar a presença potencial de agentes patogênicos $(1,5,7)$.
As doenças transmitidas por alimentos (DTA) constituem um dos problemas de saúde pública mais frequentes do mundo contemporâneo, podendo ser causadas pela ingestão de alimentos e/ ou água contaminados por microrganismos patogênicos (8). Segundo a Secretaria de Vigilância em Saúde (SVS) do Ministério da Saúde, leite e derivados estão entre os principais alimentos incriminados em surtos de DTA, de acordo com o levantamento realizado de 2007 a 2018 (9). Alguns pequenos produtores de queijo, por falta de orientação e informações, improvisam instalações deficientes para fabricação do produto, sem higienização, com armazenamento inadequado, transporte e exposição a temperaturas de risco, além de falta de controle sanitário do rebanho, que contribuem para a má qualidade higiênico-sanitária dos queijos. Nos comércios varejistas também pode haver contaminação durante a manipulação, embalagem e armazenamento sob temperatura inadequada (10).

Tendo em vista a grande importância socioeconômica do queijo, este trabalho avaliou a qualidade microbiológica de queijos Minas produzidos industrialmente e artesanalmente comercializados na cidade de Belo Horizonte, MG.

\section{MATERIAL E MÉTODO}

Para o estudo, foram coletadas 18 amostras de queijo que foram codificadas por letras do alfabeto (de A a R), sendo nove do tipo Minas frescal industrializado, comercializados em cinco supermercados, e nove do tipo Minas artesanal, comercializa- 
dos em nove lojas do Mercado Municipal, em abril de 2018, na cidade de Belo Horizonte, MG.

Durante a coleta das amostras, foi aferida a temperatura dos queijos durante a fase de exposição para venda no mercado varejista, por meio de termômetro digital infravermelho.

As amostras adquiridas foram devidamente identificadas e transportadas em caixas isotérmicas resfriadas com gelo reciclável e encaminhadas em um tempo máximo de 30 minutos para o Laboratório de Análises Microbiológicas de Alimentos do Centro Universitário UNA (LABMICRO-UNA). Os parâmetros analisados foram contagem de coliformes a $45^{\circ} \mathrm{C}$ (coliformes termotolerantes), cujo resultado é expresso em "Número Mais Provável" por grama da amostra (NMP/g), presença ou ausência de Salmonella sp. em 25 gramas da amostra e contagem de Staphylococcus coagulase-positiva, cujo resultado é expresso em Unidades Formadoras de Colônias por grama da amostra (UFC/g). A contagem de Staphylococcus coagulase-positiva é uma forma indireta de se contar Staphylococcus aureus, pois essa é a espécie do gênero mais associada a intoxicações alimentares. Existem registros na literatura de outras espécies de Staphylococcus toxigênicas e como a produção da enzima coagulase é considerada uma indicação de patogenicidade entre as espécies de Staphylococcus, ficou estabelecido no regulamento técnico sobre padrões microbiológicos para alimentos a enumeração de Staphylococcus coagulase-positiva em substituição à determinação de $S$. aureus $(11,12)$.

A metodologia utilizada para a análise seguiu o Compendium of Methods for the Microbiological Examination of Food - APHA (American Public Health Association) (13). Para a análise, $25 \mathrm{~g}$ de cada amostra foram pesadas, acrescidas de $225 \mathrm{~mL}$ de água peptonada estéril e homogeneizadas (diluição $10^{-1}$ ). A partir dessa diluição foram realizadas as diluições seriadas até $10^{-3}$. A análise de coliformes a $45{ }^{\circ} \mathrm{C}$ foi realizada por meio do método dos Tubos Múltiplos. Para tal, $1 \mathrm{~mL}$ de cada diluição foi inoculado em séries de três tubos, contendo $9 \mathrm{~mL}$ de caldo Lauril Sulfato Triptose (LST), com tubo de Durham invertido. Os tubos foram incubados a $37{ }^{\circ} \mathrm{C}$ por $24 \mathrm{~h}$. A partir dos tubos com leitura positiva foram realizados os testes para confirmação de coliformes a $45{ }^{\circ} \mathrm{C}$, inoculando, de cada tubo positivo, uma alçada para tubos contendo $10 \mathrm{~mL}$ de Caldo Escherichia coli (EC) e tubo de Durham invertido. Os tubos foram incubados a $45{ }^{\circ} \mathrm{C}$ por 24 horas. Foram considerados como positivos os tubos que apresentaram produção de gás visível ou efervescência quando agitados levemente.

A análise de Staphylococcus coagulase-positiva foi realizada com a inoculação de $0,1 \mathrm{~mL}$ de cada diluição $\left(10^{-1}\right.$ a $\left.10^{-3}\right)$ em ágar Baird Parker, utilizando o método de espalhamento em superfície. Em seguida as placas foram incubadas invertidas a 37 ${ }^{\circ} \mathrm{C}$ por 48 h. Foram consideradas as colônias negras brilhantes com bordas regulares, apresentando halo claro transparente. Para a confirmação de Staphylococcus coagulase-positiva, foram selecionadas cinco colônias típicas e atípicas de cada placa, que foram submetidas à coloração de Gram, com posterior realização das provas de catalase e o teste de coagulase livre em tubo com plasma de coelho, que consiste na comprovação da capacidade de coagular o plasma pela ação da enzima coagulase $(11,13)$.

Para a análise de Salmonella sp. foi realizado o processo de pré-enriquecimento da amostra, adicionando $25 \mathrm{~g}$ desta em $225 \mathrm{~mL}$ de água peptonada tamponada. A amostra foi homogeneizada e incubada a $35^{\circ} \mathrm{C}$ por $24 \mathrm{~h}$. A partir do pré-enriquecimento, foram inoculados $1 \mathrm{~mL}$ de cada diluição para tubos contendo $10 \mathrm{~mL}$ de caldo Tetrationato (TT) e caldo Rappaport Vassiliadis (RV) seguida de incubação dos tubos a $35^{\circ} \mathrm{C}$ por $24 \mathrm{~h}$. A partir dos caldos seletivos de enriquecimento, foi inoculada uma alçada de cada tubo em placas de ágar Xilose Lisina Desoxicolato (ágar XLD) e ágar Hektoen (ágar HE). As placas foram incubadas invertidas a $35^{\circ} \mathrm{C}$ por 48 horas. De duas a três colônias típicas de Salmonella sp. em cada meio de cultura foram semeadas em tubos inclinados de ágar Lisina Ferro (LIA) e ágar Tríplice Açúcar Ferro (TSI), incubados a $35^{\circ} \mathrm{C}$ por $24 \mathrm{~h}$. As colônias identificadas, em LIA e TSI, como Salmonella sp. foram submetidas a confirmação por meio de sorologia com soro anti-Salmonella somáticos e flagelares (13).

Todas as análises microbiológicas foram realizadas em triplicata e os resultados obtidos foram comparados com os padrões microbiológicos estabelecidos em legislação específica $(12,16)$. 
Tabela 1. Temperaturas de exposição à venda das diferentes marcas (A-R) de queijo Minas industrializado comercializado em supermercados e de queijo Minas artesanal comercializadas no Mercado Municipal de Belo Horizonte, MG, Brasil.

\begin{tabular}{|c|c|c|c|}
\hline Origem & Marcas & Temperatura durante a coleta $\left({ }^{\circ} \mathrm{C}\right)$ & Segmento de comercialização \\
\hline \multirow{9}{*}{ Industrializado* } & A & 18,8 & \multirow{9}{*}{ Supermercado } \\
\hline & B & 9,4 & \\
\hline & C & 5,7 & \\
\hline & D & 14,1 & \\
\hline & $\mathrm{E}$ & 19,6 & \\
\hline & $\mathrm{F}$ & 8,7 & \\
\hline & G & 13,8 & \\
\hline & H & 10,7 & \\
\hline & I & 13,2 & \\
\hline \multirow{9}{*}{ Artesanal $^{* *}$} & J & 24,6 & \multirow{9}{*}{ Mercado Municipal } \\
\hline & K & 20,9 & \\
\hline & L & 20,0 & \\
\hline & M & 25,4 & \\
\hline & N & 20,7 & \\
\hline & 0 & 19,8 & \\
\hline & $P$ & 23,7 & \\
\hline & Q & 21,4 & \\
\hline & R & 22,2 & \\
\hline
\end{tabular}

*Temperatura permitida por legislação $\leq 8{ }^{\circ} \mathrm{C}$ - Regulamento Técnico MERCOSUL de Identidade e Qualidade de Queijo Minas Frescal, MERCOSUL/ GMC/RES.N ${ }^{0} 145 / 96$ (14). **Temperatura permitida por legislação $\leq 10^{\circ} \mathrm{C}$ - Normas para centro de distribuição Queijo Minas Artesanal, IMA, Anexo II, portaria 818 (15). Os resultados em negrito representam amostras inadequadas com temperaturas acima da permitida pela legislação vigente.

\section{RESULTADO E DISCUSSÃO}

A Tabela 1 apresenta a temperatura em que se encontravam os queijos Minas industrializados e artesanais nos seus pontos de venda no momento da compra. Oito (88,9\%) amostras de queijo Minas frescal industrializados se encontravam em temperatura acima do permitido; as amostras artesanais, todas $(100 \%)$ estavam expostas a temperaturas inadequadas.

Os resultados referentes à contagem de coliformes a $45{ }^{\circ} \mathrm{C}$, Staphylococcus coagulase-positiva e pesquisa de Salmonella sp. nas amostras de queijo
Minas industrializado e de queijo Minas artesanal, encontram-se nas Tabelas 2 e 3, respectivamente. Duas (22\%) amostras de queijo Minas industrializado apresentaram contagens acima do limite máximo para coliformes a $45^{\circ} \mathrm{C}$ (Tabela 2 ) e quatro (44 $\%$ ) amostras de queijo Minas artesanal estavam em desacordo com os limites estabelecidos (Tabela 3). Em relação à contagem de Staphylococcus coagulase-positiva, $100 \%$ das amostras tanto de queijos industrializados quanto de queijos artesanais apresentaram contagem superior aos padrões legais estabelecidos $(12,16)$. Tanto os queijos artesanais quanto os industrializados (100\%) não apresentaram contaminação por Salmonella sp. (Tabela 2 e 3). 
Tabela 2. Presença de coliformes a $45{ }^{\circ} \mathrm{C}(\mathrm{NMP} / \mathrm{g})$, Staphylococcus coagulase positiva (UFC/g) e Salmonella sp. em queijos Minas industrializados comercializados em supermercados de Belo Horizonte, MG.

\begin{tabular}{|c|c|c|c|}
\hline Amostra & $\begin{array}{c}\text { Coliformes a } 45^{\circ} \mathrm{C} \\
\text { (NMP/g) }\end{array}$ & $\begin{array}{c}\text { Staphylococcus } \\
\text { coagulase-positiva (UFC/g) }\end{array}$ & $\begin{array}{l}\text { Salmonella sp. } \\
\qquad(\mathrm{P} / \mathrm{A})\end{array}$ \\
\hline A & $2,3 \times 10^{1}$ & $3,3 \times 10^{3}$ & Ausência \\
\hline B & $<3,0$ & $1,1 \times 10^{6}$ & Ausência \\
\hline C & $2,3 \times 10^{1}$ & $1,2 \times 10^{3}$ & Ausência \\
\hline D & $2,0 \times 10^{3}$ & $1,7 \times 10^{4}$ & Ausência \\
\hline$E$ & $2,3 \times 10^{1}$ & $3,4 \times 10^{5}$ & Ausência \\
\hline $\mathrm{F}$ & $1,5 \times 10^{1}$ & $9,0 \times 10^{4}$ & Ausência \\
\hline G & $<3,0$ & $6,0 \times 10^{2}$ & Ausência \\
\hline $\mathrm{H}$ & $<3,0$ & $2,2 \times 10^{4}$ & Ausência \\
\hline I & $1,1 \times 10^{4}$ & $1,1 \times 10^{6}$ & Ausência \\
\hline Variação & $<3,0$ a $1,1 \times 10^{4}$ & $6,0 \times 10^{2}$ a $1,1 \times 10^{6}$ & \\
\hline Padrão legal* & Máximo $5 \times 10^{2}$ & Máximo $5 \times 10^{2}$ & Ausência \\
\hline
\end{tabular}

${ }^{*} \mathrm{RDC} \mathrm{n}^{0} 12$, de 2 de janeiro de 2001 (12). 0s resultados em negrito representam amostras inadequadas com contagem acima da permitida pela legislação vigente. NPM/g: Número mais provável/grama. UFC/g: Unidades formadoras de colônias/grama. P/A: presença ou ausência.

Tabela 3. Presença de coliformes a $45{ }^{\circ} \mathrm{C}$ (NMP/g), Staphylococcus coagulase positiva (UFC/g) e Salmonella sp. em queijos Minas artesanais comercializados no Mercado Municipal de Belo Horizonte, MG.

\begin{tabular}{|c|c|c|c|}
\hline Amostra & $\begin{array}{l}\text { Coliformes a } 45^{\circ} \mathrm{C} \\
(\mathrm{NMP} / \mathrm{g})\end{array}$ & $\begin{array}{c}\text { Staphylococcus } \\
\text { coagulase-positiva (UFC/g) }\end{array}$ & $\begin{array}{l}\text { Salmonella sp. } \\
\text { (P/A) }\end{array}$ \\
\hline J & $2,0 \times 10^{3}$ & $2,8 \times 10^{5}$ & Ausência \\
\hline $\mathrm{K}$ & $9,0 \times 10^{3}$ & $2,1 \times 10^{5}$ & Ausência \\
\hline$L$ & $2,0 \times 10^{1}$ & $5,2 \times 10^{5}$ & Ausência \\
\hline M & 9,0 & $1,8 \times 10^{5}$ & Ausência \\
\hline $\mathrm{N}$ & $5,0 \times 10^{2}$ & $2,1 \times 10^{4}$ & Ausência \\
\hline 0 & $2,0 \times 10^{3}$ & $7,0 \times 10^{3}$ & Ausência \\
\hline$P$ & $<3,0$ & $7,0 \times 10^{3}$ & Ausência \\
\hline Q & $2,0 \times 10^{2}$ & $3,3 \times 10^{4}$ & Ausência \\
\hline $\mathrm{R}$ & $9,0 \times 10^{3}$ & $4,6 \times 10^{6}$ & Ausência \\
\hline Variação & $<3,0$ a $9,0 \times 10^{3}$ & $2,1 \times 10^{4}$ a $4,6 \times 10^{6}$ & \\
\hline Padrão legal ${ }^{*}$ & Máximo $5 \times 10^{2}$ & Máximo $1 \times 10^{3}$ & Ausência \\
\hline
\end{tabular}

* Lei $n^{\circ}$ 14.185, de 31 de janeiro de 2002 (16). Os resultados em negrito representam amostras inadequadas com contagem acima da permitida pela legislação vigente. NPM/g: Número mais provável/grama. UFC/g: Unidades formadoras de colônias/grama. P/A: presença ou ausência. 
Levando em consideração que as dezoito (100 \%) amostras de queijo artesanal e industrializado estavam em desacordo com a legislação vigente, em pelo menos um dos parâmetros nela estabelecidos, todas foram consideradas impróprias para o consumo. Sendo assim, foi calculado o percentual de amostras em acordo ou em desacordo com os padrões legais para cada análise microbiológica realizada (Tabela 4).
As contagens microbianas foram semelhantes para os dois tipos de queijo avaliados, com exceção da contagem de coliformes a $45{ }^{\circ} \mathrm{C}$, em que os queijos artesanais apresentaram o dobro de amostras fora dos padrões legais quando comparadas às amostras de queijos industrializados (Tabela 4).

Os parâmetros estabelecidos em relação a temperatura de conservação são imprescindíveis na avaliação da qualidade dos alimentos, devido aos

Tabela 4. Número de amostras e percentual de Queijo Minas industrializado e artesanal comercializados, respectivamente, em supermercados e no Mercado Municipal de Belo Horizonte, MG, quanto aos padrões microbiológicos legais para contagem de coliformes a $45{ }^{\circ} \mathrm{C}$, contagem de Staphylococcus coagulase positiva (SCP) e pesquisa de Salmonella sp.

\begin{tabular}{|c|c|c|c|c|c|}
\hline \multirow{2}{*}{ Queijo Minas } & \multicolumn{2}{|c|}{ Dentro dos padrões } & \multicolumn{2}{|c|}{ Fora dos padrões } \\
\cline { 2 - 6 } & Contaminante & N & $\%$ & N & $\%$ \\
\hline \multirow{2}{*}{ Industrializados* } & Coliformes a $45^{\circ} \mathrm{C}$ & 7 & 78 & 2 & 22 \\
\hline & SCP & 0 & 0 & 9 & 100 \\
\hline & Salmonella sp. & 9 & 100 & 0 & 0 \\
\hline Artesanais** & Coliformes a $45^{\circ} \mathrm{C}$ & 5 & 56 & 4 & 44 \\
\hline & SCP & 0 & 0 & 9 & 100 \\
\hline & Salmonella sp. & 9 & 100 & 0 & 0 \\
\hline
\end{tabular}

* $\operatorname{RDC} n^{\circ}$ 12, de 2 de janeiro de 2001 (12). ** Lei n 14.185, de 31 de janeiro de 2002 (16). N: número de amostras.

riscos que podem apresentar à saúde $(14,15)$. A temperatura ocupa lugar de proeminência dentre os inúmeros parâmetros extrínsecos que influenciam a multiplicação dos microrganismos em alimentos. $\mathrm{O}$ queijo Minas possui uma alta umidade, que aliada ao binômio tempo e temperatura, pode favorecer a proliferação de microrganismos (1). Nesse estudo, apenas uma $(5,5 \%)$ dentre todas as amostras avaliadas encontrava-se em temperatura adequada de conservação. O fato de a maioria dos queijos avaliados estar exposta à venda sob temperaturas inadequadas pode ter contribuído para as altas contagens microbianas encontradas.

Os coliformes totais e termotolerantes estão frequentemente presentes no leite cru e são destruídos após a pasteurização $(17,18)$. As altas contagens de coliformes termotolerantes encontradas em quatro (44 \%) amostras de queijo Minas artesanal podem estar relacionadas à ausência de pasteurização e às más condições de higiene durante a ordenha e distribuição do produto aos clientes. Em contrapartida, na produção dos queijos industrializados é utilizado, como matéria-prima, o leite pasteurizado. Entretanto, as altas contagens de coliformes termotolerantes encontradas nesse tipo de queijo podem indicar uma recontaminação após a pasteurização, devido à falta de adequação às Boas Práticas de Fabricação (BPF) $(19,20)$.

Conforme os resultados das análises de queijos Minas realizadas no presente trabalho, duas (22\%) das amostras industrializadas apresentaram-se fora dos padrões para esse grupo de microrganismos, o que indica falhas pós-processamento em alimentos pasteurizados, pois os coliformes são facilmente destruídos por elevadas temperaturas (21). 
A contagem de Staphylococcus coagulase-positiva em alimentos com valores de $10^{3}$ a $10^{4} \mathrm{UFC} / \mathrm{g}$ é apontada como uma ameaça à saúde pública, e quando os valores de referência são próximos a $10^{5}$ UFC/g torna-se uma ameaça epidemiológica pela probabilidade da existência de enterotoxinas, que em quantidade significativa causam intoxicação caso a cepa de Staphylococcus spp. seja toxigênica (22). De fato, é preocupante que três (33\%) das amostras industrializadas apresentaram valores $\geq$ $10^{5} \mathrm{UFC} / \mathrm{g}$ e cinco $(55 \%)$ das amostras artesanais seguiram o mesmo padrão. Em um estudo realizado com queijos Minas comercializados do Estado de Goiás, $70 \%$ das amostras de queijos artesanais e industrializados estavam fora dos padrões de referência para Staphylococcus coagulase-positiva (19). A ocorrência foi observada também em queijos do tipo coalho $(72,7 \%)$ e em queijos do tipo manteiga $(84,7 \%)$, produzidos e comercializados no Estado do Rio Grande do Norte (23).

A bactéria $S$. aureus é frequentemente encontrada na nasofaringe do ser humano e pode, a partir desses locais, alcançar outra parte da pele ou mucosa e facilmente contaminar os alimentos, por meio do contato com as mãos (24). Essas bactérias são resistentes à elevada concentração de sal e são capazes de produzir enterotoxinas entre $10{ }^{\circ} \mathrm{C}$ e 46 ${ }^{\circ} \mathrm{C}$. Desta forma, a presença desse microrganismo, sobretudo em alimentos processados submetidos a tratamento térmico, é um risco à saúde do consumidor, uma vez que mesmo as bactérias sendo eliminadas, as suas toxinas são termoestáveis, permanecendo ativas e passíveis de causar intoxicação alimentar $(25,26)$.

Dentre os surtos mais comuns de intoxicação alimentar estão a intoxicação estafilocócica causada pela bactéria $S$. aureus (27) e uma das formas de prevenção dessa intoxicação é a higienização de forma apropriada das mãos de manipuladores de alimentos com água corrente e sabonete líquido aliado ao uso de antisséptico $(28,29)$.

A Salmonella sp. é um dos agentes microbianos mais comuns em surtos de DTA no Brasil, e sua presença em alimentos é considerada um relevante problema de saúde pública. Pode estar relacionada a diferentes fontes, porém, a pasteurização é capaz de eliminá-la. No queijo Minas frescal, a contaminação pode estar relacionada à ineficiência da pasteurização, manipuladores portadores do microrganismo ou à utilização de água não potável no processamento $(9,30)$. Os resultados microbiológicos neste estudo revelaram ausência de Salmonella sp. em $100 \%$ das amostras analisadas, em concordância com a legislação vigente. Esse resultado foi semelhante em diversos estudos, como o realizado em Goiás que analisou o queijo Minas obtidos de leite cru e pasteurizado, e nenhuma amostras apresentou contaminação por essa bactéria (19).

A Lei Estadual $n^{\circ} 14.581$, de 17 de janeiro de 2003, dispõe sobre o processo da produção de queijo Minas artesanal, determinando as exigências para legalização com relação aos parâmetros microbiológicos, sanitários, higiênicos, cadastramento no Instituto Mineiro de Agropecuária (IMA), água, equipamentos, rotulagens, transporte e comercialização (16). Contudo, muitos produtores de queijo rurais ainda não são legalizados, o que expõe os consumidores a um risco microbiológico (16).

A presença de selos de inspeção, como o SIF (Selo de Inspeção Federal), em alimentos indica ao consumidor que existe fiscalização na produção, por parte de agentes de inspeção sanitária como o Ministério da Agricultura, Pecuária e Abastecimento (MAPA). Quando o produto não apresenta qualquer tipo de selo, como no caso das amostras de queijo avaliadas nesse estudo, significa que ele não recebe inspeção de órgãos governamentais (19).

\section{CONCLUSÃO}

Com base nos resultados, a qualidade higiênico-sanitária de $100 \%$ das amostras de queijo Minas, adquiridos em supermercados e Mercado Municipal de Belo Horizonte, MG, estava insatisfatória. Todas as amostras apresentaram contagens de Staphylococcus coagulase-positiva acima dos padrões legais. Em relação aos coliformes a $45{ }^{\circ} \mathrm{C}$, indicadores de patógenos entéricos, $22 \%$ das amostras de queijo Minas industrializado e 44\% das amostras de queijo Minas artesanal estavam fora do valor aceitável. Diante dos riscos à saúde associados a um produto vastamente consumido e reconhecido no país, torna-se urgente o cumprimento das nor- 
mas legais para a fabricação de queijos, sejam eles elaborados a partir de leite pasteurizado em escala industrial ou elaborados de forma artesanal a partir de leite cru. É de extrema importância a regulari- dade da prática de treinamento dos manipuladores, assim como uma fiscalização efetiva desde a produção dos queijos até a sua exposição no mercado varejista.

\section{REFERÊNCIAS}

1. Sobral D; Costa RGB; Paula JCJ; Teodoro VAM; Moreira GMM; Pinto MS. Principais defeitos em queijo minas artesanal: Uma revisão. Rev. Inst. Latic. Cândido Toste. 2017;72:108-120. DOI: 10.14295/2238-6416. v72i 2.600

2. Arruda MLT; Nicolau ES; Reis AP; Araujo AS; Mesquita AJ. Ocorrência de Staphylococcus coagulase positiva em queijos Minas tipos frescal e padrão comercializados nas feiras livres de Goiânia-GO. Rev. Inst. Adolfo Lutz. 2007;66(3):292-298.

3. Ahagon CM, Mattos EC, Nascimento AL, Daros VSMG, Col RD. Avaliação da qualidade de queijo Minas frescal quanto aos ensaios de umidade, gordura e presença de matérias estranhas. Revista saúde.com. 2017; 13(3): 956-964. DOI: 10.22481/rsc.v13i3i.474

4. Machado EC; Ferreira CLLF; Fonseca LM; Soares FM; Pereira Junior FN. Características físico-químicas e sensoriais do queijo Minas artesanal produzido na região do Serro, Minas Gerais. Ciênc. tecnol. aliment. 2004;24(4):516-521. DOI: 10.1590/S0101-20612004000 400006 .

5. Silva D; Trindade R; Reis C; Cabezas S; Fonseca E. Ocorrência de Listeria monocytogenes em queijo do tipo Minas frescal comercializado na cidade de Barra Mansa, RJ. Infarma. 2011;23(1/2):60-64.

6. Carvalho JDG; Viotto; WH; Kuaye; AY. The quality of Minas frescal cheese produced by different technological processes. Food Control. 2007;18:262-267. DOI: 10.1016/j.foodcont.2005.10.005

7. Salvador M; Camassola M; Moschen ES; Zanrosso AV. Avaliação microbiológica de queijo prato e parmesão ralado. Bol. Cent. Pesq. Process. Aliment. 2001;1:65-74. DOI: $10.5380 /$ cep.v19i1.1223.

8. Welker CAD; Both JMC; Longaray SM. Análise microbiológica dos alimentos envolvidos em surtos de doenças transmitidas por alimentos (DTA) ocorridos no estado do Rio Grande do Sul, Brasil. Rev. Bras. Biociências. 2010; 8: 44-48.

9. BRASIL. Surtos de Doenças Transmitidas por Alimentos no Brasil. Secretaria de Vigilância em Saúde. Ministério da Saúde. Disponível em http://portalarquivos.saude. gov.br/images/pdf/2017/maio/29/Apresentacao-SurtosDTA-2017.
10. Sartori D; Sallotti-Souza BM. Utilização de embalagem com atmosfera modificada e uso de irradiação ionizante para a conservação de queijos. RCMV-UNORP. 2017; 1:16-22.

11. Silva N; Junqueira VCA; Silveira; NFA; Taniwaki; MH; Gomes RAR; Okazaki; MM. Manual de métodos de análise microbiológica de alimentos e água. 5. ed. São Paulo: Varela; 2017.

12. BRASIL. Regulamento técnico sobre padrões microbiológicos para alimentos. Resolução RDC $\mathrm{n}^{\circ} 12$, de 2 de janeiro de 2001. Ministério da Saúde. Agência nacional de vigilância sanitária (ANVISA). Diário Oficial [da] República Federativa do Brasil, Brasília, DF, 10 janeiro de 2001. Seção 1.

13. APHA. Compendium of Methods of Microbiological Examination of Foods. 5th ed. Salfinger Y; Tortorello; ML editors. American Public Health Association, Washington DC; 2015. p. 21-23. APUD Resolução $\mathrm{RDC} \mathrm{n}^{\circ} 12$, de 2 de janeiro de 2001). Diário Oficial [da] República Federativa do Brasil, Brasília, DF, 10 janeiro de 2001. Seção 1.

14. BRASIL. Regulamento Técnico de Identidade e Qualidade de Queijo Minas Frescal. RDC n 145 de 13 de dezembro de 1996. MERCOSUL/GMC.

15. IMA, Instituto Mineiro de Agropecuária. Normas para centro de distribuição queijo Minas artesanal. Superintendência de Segurança Alimentar e Certificação. Anexo II, portaria 818 de 12 de dezembro de 2006.

16. MINAS GERAIS. Dispõe sobre o processo de produção de queijo Minas artesanal e dá outras providências. Lei 14.185, de 31 de janeiro de 2002. [citado em 28 de agosto de 2018]. Disponível em: https://leisestaduais. com.br/mg/lei-ordinaria-n-14185-2002-minas-geraisdispoe-sobre-o-processo-de-producao-do-queijo-minasartesanal-e-da-outras-providencias.

17. Leite Júnior AFS; Florentino ER; Oliveira EB; Suelândia $\mathrm{N}$; Torrano ADM. Qualidade microbiológica de queijo coalho comercializado à temperatura ambiente ou sob refrigeração, em Campina Grande-PB. Hig. Aliment. 2000;14:53-59.

18. Quintana RC; Carneiro LC. Avaliação das condições higiênico-sanitárias dos queijos minas frescal e mussarela produzidos na cidade de Morrinhos - GO. Rev. Bras. Saúde Prod. An. 2007;8(3):205-211. 
19. Dias BF; Ferreira SM; Carvalho VS; Soares DSB. Qualidade microbiológica e físico-química de queijo minas frescal artesanal e industrial. Agric Neotrop. 2016;3(3):57-64

20. Perry KSP. Queijos: aspectos químicos, bioquímicos e microbiológicos. Quím. Nova. 2004;27(2):293-300. DOI: $10.1590 / \mathrm{S} 0100-40422004000200020$.

21. Carvalho LR; Magalhães JT. Avaliação da qualidade microbiológica dos caldos de cana comercializados no centro de Itabuna-BA e práticas de produção e higiene de seus manipuladores. Rev. Baiana Saúde Pública. 2007; 31(2):238-245.

22. Ferrari RG; Winkler SM; Oliveira TCRM. Avaliação microbiológica de alimentos isentos de registro no Ministério da Saúde. Semina: Ciênc Agrárias. 2007; 28 (2):241-250

23. Feitosa T; Borges MF; Nassu RT; Azevedo EHF; Muniz CR. Pesquisa de Salmonella sp., Listeria sp. e microrganismos indicadores higiênico sanitário em queijos produzidos no Estado do Rio Grande do Norte. Ciênc. Tecnol. Aliment. 2003;23:162-165. DOI: 10.1590/ S0101-20612003000400030.

24. Cassettari VC; Strabelli T; Medeiros EAS. Staphylococcus aureus bacteremia: what is the impact of oxacillin resistance on mortality? Braz. J. Infect. Dis. 2005; 9(1):70-76. DOI: 10.1590/S1413-86702005000100012.
25. Cunha NA; Silva CGM; Stamford TLM. Staphylococcus enterotoxigênicos em alimentos in natura e processados no estado de Pernambuco, Brasil. Ciênc. Tecnol. Aliment. 2002; 22(3):263-271. DOI: 10.1590/S010120612002000300012 .

26. Ferrasso MM; Gonzalez HL; Timm CD. Staphylococcus hyicus. Arq Inst Biol. São Paulo. 2015;82:00401. DOI: 1590/1808-1657000672013.

27. Ferreira GB; Oliveira ACS; Marson JM; Terra APS. Pesquisa de Staphylococcus aureus em queijos tipo minas frescal comercializados na região do triângulo mineiro. Rev. Baiana Saúde Pública. 2010;34(3): 575-589. DOI: 10.22278/2318-2660.2010.

28. Franco BDGM; Landgraf M. Microbiologia dos Alimentos. São Paulo: Atheneu. 2008;4 ed: 182p.

29. Kochanski S; Pierozan MK; Mossi AJ; Treichel H; Cansian RL; Ghisleni CP; Toniazzo G. Avaliação das condições microbiológicas de uma unidade de alimentação e nutrição. Aliment Nutr. 2009; 20(4): 663-668.

30. Fai AEC; Figueiredo EAT; Verdin SEF; Pinheiro NMS; Braga ARC; Stamford TLM. Salmonella spp e Listeria monocytogenes em presunto suíno comercializado em supermercados de Fortaleza (CE, Brasil): fator de risco para a saúde pública. Ciênc Saúde Coletiva. 2011;16(2):657-662. DOI: 10.1590/S141381232011000200029 . 